\title{
Posted Workers in Österreich: Grenzüberschreitende Entsendearbeit im Spannungsfeld von offenen Märkten und (supra-)nationaler Regulierung
}

\author{
Torben Krings
}

(C) Der/die Autor(en) 2019

Zusammenfassung Seit der Arbeitsmarktöffnung 2011 ist nicht nur ein starker Anstieg von individueller Arbeitsmigration nach Österreich zu verzeichnen, sondern auch von arbeitgebergesteuerter Dienstleistungsmobilität. Dieser Artikel untersucht die Entwicklung und die Regulierung von grenzüberschreitender Entsendearbeit am Beispiel der Bauwirtschaft. Ein Anstieg von Entsendungen hat nicht nur eine Nachfrage nach „flexiblen“ Arbeitskräften bedient, sondern war auch von Sorgen um einen Unterbietungswettbewerb begleitet. Mit dem Lohn- und Sozialdumping-Bekämpfungsgesetz wurde ein relativ umfassendes Regulierungsregime etabliert, welches neue Möglichkeiten zur Durchsetzung von Arbeitsstandards geschaffen hat. Die empirischen Befunde dieses Artikels legen allerdings nahe, dass dieses Regime in einem transnationalen Mobilitätsraum, der von erheblichen Ungleichheiten geprägt ist, an seine nationalen Grenzen stößt. In dieser Hinsicht stehen die Erfahrungen von Österreich stellvertretend für die Herausforderungen, mit denen vor allem kontinentaleuropäische Wohlfahrtsstaaten in der erweiterten EU konfrontiert sind, in der die soziale und institutionelle Vielfalt zugenommen hat.

Schlüsselwörter Entsendearbeit · Transnationaler Mobilitätsraum · Soziale Ungleichheit · Arbeitsstandards · Regulierung

\section{Posted workers in Austria: cross-border mobile labour between open markets and (supra-)national regulation}

Abstract Since the opening of the labour market in 2011, Austria experienced a huge rise not only in individual migration but also in employer-led service mo-

T. Krings $(\bowtie)$

Institut für Soziologie, Johannes Kepler Universität Linz, Altenberger Straße 69, 4040 Linz,

Österreich

E-Mail: torben.krings@jku.at 
bility. This article examines the development and regulation of posted labour in the construction industry. It shows how an increase in worker posting met a demand for 'flexible' labour while also raising concerns about a race to the bottom. In response to such concerns the 'Anti-Wage and Social Dumping Act' was established which represents a rather comprehensive regime of labour market regulation. However, the results of the article suggest that such a nationally orientated regime reaches its limits in a transnational mobility space which is characterised by huge inequalities. Thus, the Austrian experience is representative of the challenges which continental welfare states have to face in an enlarged EU with increased social and institutional diversity.

Keywords Posted labour · Transnational mobility space $\cdot$ Social inequality · Employment standards $\cdot$ Regulation

\section{Einleitung}

Im Sommer 2017 empfing der damalige österreichische Bundeskanzler Christian Kern den französischen Präsidenten Emmanuel Macron zu einem „Sozialgipfel“, dessen Schwerpunkt eine Reform der EU-Entsenderichtlinie war. Dieser Gipfel, zu dem auch die tschechischen und slowakischen Regierungschefs Robert Fico und Bohuslav Sobotka geladen waren, signalisierte zweierlei: Zum einen ist die zwischenstaatliche, intergouvernementale Ebene weiterhin von großer Bedeutung für die Aushandlung von EU-Policy-Reformen, ungeachtet von Bestrebungen der Europäischen Kommission, hier die Initiative zu ergreifen. Zum anderen dokumentierte dieser Gipfel die Bedeutung, welche grenzüberschreitende Entsendearbeit mittlerweile in Österreich und anderswo erlangt hat. Dies trifft insbesondere auf Entsendungen aus den neuen EU-Mitgliedsstaaten (NMS) zu.

Die Öffnung des österreichischen Arbeitsmarktes 2011 und $2014^{1}$ hat eine neue Dynamik der Arbeitsmobilität ausgelöst. Seitdem ist nicht nur ein starker Anstieg von individueller Migration zu beobachten (vgl. hierzu die Beiträge im ÖZS-Sonderheft 4/2016), sondern auch von arbeitgebergesteuerter Dienstleistungsmobilität. Letztere hat noch keine vergleichbare Beachtung in der sozialwissenschaftlichen Migrationsforschung gefunden, obwohl sie wichtige Fragen zur Regulierung von Arbeit und Beschäftigung in einem transnationalen Mobilitätsraum aufwirft (Bosch 2013, S. 279). Eine dieser Fragen ist, wie die grenzüberschreitende Erbringung von Dienstleistungen erfolgen kann, ohne dass nationale Arbeits- und Sozialstandards unterlaufen werden (Dølvik et al. 2014; Krings 2016). Vor allem für Hochlohnländer wie Österreich stellt die zeitlich befristete Entsendung von Arbeitskräften (posted workers) eine Herausforderung dar, nicht zuletzt, weil sie immer wieder mit Fäl-

\footnotetext{
1 Österreich hatte 2004 für acht EU-Beitrittsländer aus Mittel- und Osteuropa nicht nur die Arbeitnehmerfreizügigkeit eingeschränkt, sondern auch die Dienstleistungsfreiheit etwa im Baugewerbe. Diese Übergangsbestimmungen wurden auch für Bulgarien und Rumänien erlassen, die 2007 der EU beitraten, ebenso für Kroatien, welches 2013 beitrat.
} 
len von Lohn- und Sozialdumping in Verbindung gebracht wird (Die Presse 2017; Szigetvari 2018a).

Dieser Artikel untersucht den Anstieg und die Regulierung von grenzüberschreitender Entsendearbeit am Beispiel der österreichischen Bauwirtschaft. Folgende Forschungsfragen stehen im Mittelpunkt der Analyse: Welches sind die Gründe für den Anstieg von Entsendearbeit? Wie hat sich diese Mobilität auf den Bauarbeitsmarkt ausgewirkt? Welche Bestrebungen hat es gegeben, Entsendearbeit wieder in nationale Arbeitsbeziehungen ,einzubetten“? Welche Schwierigkeiten sind in diesem Zusammenhang zu beobachten?

Der Beitrag ist wie folgt aufgebaut: Zuerst wird der Untersuchungsgegenstand in einen theoretisch-konzeptionellen Kontext eingeordnet. Anschließend wird kurz das methodische Vorgehen skizziert, ehe dann der Anstieg von Entsendungen seit der Arbeitsmarktöffnung nachgezeichnet wird. In den dann folgenden Abschnitten wird auf der Basis von ExpertInneninterviews gezeigt, dass Arbeitsmarktakteure in der Bauwirtschaft mit verschiedenen ,Varianten des Lohndumpings“ konfrontiert sind. Um ein Unterlaufen von Arbeitsstandards zu unterbinden, wurde mit dem Lohn- und Sozialdumping-Bekämpfungsgesetz ein relativ umfassendes Regulierungsregime geschaffen. Allerdings legen die Ergebnisse dieses Beitrags nahe, dass dieses Regime sowohl in sozialer als auch in räumlicher Hinsicht an seine Grenzen stößt.

\section{Entsendearbeit in einem transnationalen Mobilitätsraum}

\subsection{Dienstleistungsmobilität zwischen Arbeitsort- und Herkunftslandprinzip}

Entsendungen finden im Rahmen der EU-Dienstleistungsfreiheit statt, die eine der vier Grundfreiheiten des europäischen Binnenmarktes ist. Dabei steht die Genese der Dienstleistungsfreiheit paradigmatisch für einen Prozess der europäischen Integration, der sich in einem Spannungsfeld zwischen nationaler Öffnung und (supra-)nationaler Re-Regulierung bewegt. Die Schaffung eines Binnenmarktes war von einer Logik der „negativen Integration“ (Scharpf 1998) und dem Niederreißen von Marktbarrieren geprägt. Im Zusammenhang mit der grenzüberschreitenden Erbringung von Dienstleistungen bedeutete dies die Anwendung des EU-Wettbewerbsrechts, welches Auswirkungen auf die Regulierung von Mobilität und Beschäftigung hatte. Während bei der Arbeitnehmerfreizügigkeit traditionell das Arbeitsortprinzip (lex loci laboris) zur Anwendung kommt, dominierte bei der Dienstleistungsfreiheit zumindest bis in die 1990er-Jahre das Herkunftslandprinzip (Bosch 2013, S. 280-281). In dieser Hinsicht steht grenzüberschreitende Entsendearbeit beispielhaft für eine ,institutionelle Entbettung“ (Holst 2015) von Arbeit, die aus dem räumlichen Geltungsbereich des Ziellandes herausgelöst wird.

Gleichzeitig steht die Auseinandersetzung um Entsendearbeit aber auch für Ansätze einer „Wiedereinbettung“ von Marktkräften, wobei hier eine Verschränkung der nationalen und der supranationalen Ebene zu beobachten ist. In den 1990erJahren gewann die Frage, wie Dienstleistungsmobilität angesichts eines Anstiegs von Entsendungen reguliert werden sollte, an Bedeutung. Nachdem eine europaweite Regelung zunächst am Widerstand von Entsendeländern wie Portugal und dem 
Vereinigten Königreich scheiterte, reagierten einige Zielländer mit einer Reihe von nationalen Entsenderegelungen. Dies eröffnete wiederum den Raum für einen Kompromiss auf Gemeinschaftsebene, sodass 1996 die EU-Entsenderichtlinie 96/71/EG verabschiedet werden konnte. Diese Richtlinie legt einen regulatorischen Rahmen für allgemeinverbindliche Mindestnormen wie Mindestlöhne und Höchstarbeitszeiten fest, der sich am Arbeitsortprinzip orientiert (Eichhorst 1999).

Bei der Umsetzung der Entsenderichtlinie zeigten sich erhebliche nationale Variationen, welche die unterschiedlichen Traditionen der Arbeitsbeziehungen in der EU widerspiegeln. Während Österreich und Frankreich weitgehend das Equal Pay-Prinzip per Gesetz festschrieben, wurde in Deutschland neben einigen Mindestnormen nur ein allgemeinverbindlicher tarifvertraglicher Mindestlohn für den Bausektor festgelegt. In Schweden und in Dänemark wiederum wurden keine allgemeinverbindlichen Lohnstandards festgelegt, da man auf die Selbstregulierung des Arbeitsmarktes durch Kollektivverträge und die Rolle der Sozialpartner setzte (Dølvik et al. 2014, S. 151-152). ${ }^{2}$

Obwohl entsandte Arbeitskräfte in Ländern wie Österreich Anspruch auf das kollektivvertragliche Entgelt haben, befinden sie sich auch hier in einer rechtlichen Sonderstellung, da in Teilen weiterhin das Arbeitsrecht des Herkunftslandes gilt (Wiesinger 2015, S. 5-6). Auch werden entsandte Beschäftigte in der Regel nicht in die sozialen Sicherungssysteme des Ziellandes integriert, eine Tatsache, der insbesondere in kontinentaleuropäischen Wohlfahrtsstaaten eine besondere Bedeutung zukommt (s. unten). ${ }^{3}$ Zudem wird der prekäre Charakter von Entsendearbeit dadurch verschärft, dass oftmals eine Diskrepanz zwischen formalen Arbeitsstandards und tatsächlicher Durchsetzung dieser zu beobachten ist (Staples et al. 2013). Als Folge entstehen transnationale „, spaces of exception“ (Wagner und Lillie 2014), in denen entsandte Beschäftigte zwar in physischer Hinsicht am Arbeitsort präsent, in sozialer und in rechtlicher Hinsicht aber nur eingeschränkt Teil nationaler Beschäftigungssysteme sind.

\subsection{Angebot und Nachfrage in einem transnationalen Mobilitätsraum}

Im Rahmen der EU-Dienstleistungsfreiheit werden Beschäftigte eines Unternehmens für einen begrenzten Zeitraum in einen anderen Mitgliedsstaat entsandt, um dort eine entsprechende wirtschaftliche Tätigkeit für ein anderes Unternehmen zu erbringen. ${ }^{4}$ Somit ist bei dieser Form von ,,atypischer“ Arbeitsmobilität die Rolle von Firmen zentral, nicht nur im Hinblick auf die Nachfrageseite, sondern auch im Hinblick auf die Angebotsseite.

\footnotetext{
2 Dieses skandinavische Modell geriet durch eine Reihe von kontroversen Urteilen des Europäischen Gerichtshofs (,Laval-Quartett“) unter Druck und musste entsprechend nachjustiert werden (Dølvik et al. 2014, S. 174-177).

3 Gemäß der EU-Verordnung 883/2004 zur Koordinierung der Systeme der sozialen Sicherheit bleiben entsandte Beschäftigte in ihrem Herkunftsland versichert, solange die Entsendung kürzer als 24 Monate ausfällt (was der Regelfall ist).

4 Unter die Dienstleistungsfreiheit fallen auch selbstständig Erwerbstätige, die eine zeitlich befristete Tätigkeit im Ausland erbringen. Hiervon ist die Niederlassungsfreiheit zu unterscheiden, bei der eine dauerhafte selbstständige Erwerbstätigkeit in einem anderen Mitgliedsstaat angenommen wird.
} 
Die Bedeutung der Nachfrageseite für Arbeitsmigration wurde bereits von der Theorie des dualen Arbeitsmarktes herausgearbeitet (Piore 1979). Dieser Ansatz unterscheidet zwischen einem primären Teilarbeitsmarkt mit ,guten“ sicheren Jobs und einem sekundären Teilarbeitsmarkt mit „schlechten“ instabilen Jobs. Die Beobachtung von Piore (1979, S. 54-58), dass insbesondere temporäre MigrantInnen im sekundären Segment beschäftigt werden, da ihr „Referenzrahmen“ das Herkunftsland bleibt, ist eine wichtige Erkenntnis in Bezug auf Entsendearbeit, die per definitionem zeitlich befristet ist. Auch kommt der dualen Theorie das Verdienst zu, die Bedeutung von Arbeitgebern als einen ,strategischen Faktor“ (Piore 1979) für Migrationsbewegungen erkannt zu haben. Während sich Piores Ansatz noch weitgehend auf einer makrotheoretischen Ebene bewegt, haben jüngere nachfrageorientierte Ansätze den Fokus auf die Mikroebene von Firmen gelegt und herausgearbeitet, wie deren Beschäftigungsstrategien unterschiedliche Formen transnationaler Arbeitsmobilität auslösen, sei es als individuelle Migration oder arbeitgebergesteuerte Entsendearbeit (Bommes et al. 2004; Fellini et al. 2007).

Segmentationstheoretische Ansätze haben die zentrale Rolle von Firmen für die Strukturierung von Arbeitsmarktprozessen hervorgehoben, ohne zu vernachlässigen, dass die Nachfrageseite nicht losgelöst von einem sich verändernden Angebot an Arbeitskräften betrachtet werden kann (Grimshaw et al. 2017; Rubery 2007). Diese Beobachtung trifft nicht nur auf nationale Arbeitsmärkte zu, sondern auch auf einen transnationalen Mobilitätsraum wie dem der EU, wo der Arbeitsmarktzugang nicht mehr nationalstaatlich reguliert ist. Spätestens nach Ablauf einer Übergangsfrist können sich Arbeitskräfte und Dienstleister aus einem EU-Mitgliedsland anderswo frei anbieten, unabhängig davon, ob eine Knappheit an Arbeitskräften vorliegt oder nicht. Dies schafft wiederum neue Gelegenheiten für Firmen in den Zielländern dieser Mobilität, neue Beschäftigungsstrategien zu entwickeln, die vor allem auf eine Senkung der Arbeitskosten abzielen (Hunger 2000, S. 199). Somit können sich Angebot und Nachfrage bei der grenzüberschreitenden Erbringung von Dienstleistungen in einem dynamischen Verhältnis zueinander entwickeln und eine disruptive Wirkung in lokalen Arbeitsmärkten entfalten (Bosch 2013).

Eine besondere Bedeutung kommt der Rolle von transnationalen Subunternehmerketten zu, über die oftmals der Marktzugang für Entsendefirmen erfolgt. Grundsätzlich ist die Auslagerung einzelner Dienstleistungen keine neue Erscheinung in der Bauwirtschaft, die schon immer sehr arbeitsteilig organisiert war. Allerdings ist seit den 1990er-Jahren eine Bedeutungszunahme von cross-border subcontracting zu beobachten, die vor allem durch die EU-Dienstleistungsfreiheit ermöglicht wird (Bosch et al. 2013, S. 172). Grundsätzlich kann bei der Auslagerung von Dienstleistungen zwischen einer Form des ,,kooperativen Subcontracting“ und einer Form des „kompetitiven Subcontracting“ (Harvey 2003) unterschieden werden. Während Erstere auf eine Spezialisierung von Arbeitsaufgaben und beruflichem Wissen abzielt, bezweckt Letztere vor allem eine Kostensenkung. Insbesondere die kompetitive Variante des Subcontracting hat im Zuge der EU-Erweiterungen eine neue Dynamik entfaltet, indem ein verändertes Angebot an kostengünstigen Dienstleistungen auf eine entsprechende Nachfrage in der europäischen Bauwirtschaft trifft. 


\subsection{Eine neue Dynamik der Dienstleistungsmobilität in der europäischen Bauwirtschaft}

Die Erweiterungsrunden 2004 und 2007 (und auch 2013) haben den sozio-ökonomischen Kontext für innereuropäische Mobilität grundlegend verändert. Noch Mitte der 1990er-Jahre, als die Entsenderichtlinie verabschiedet wurde, betrug das Verhältnis zwischen den höchsten und den niedrigsten Durchschnittslöhnen in der EU 3 zu 1 (Belgien vs. Portugal). Mittlerweile hat sich dieses Verhältnis auf über $7 \mathrm{zu} 1$ erhöht (Niederlande vs. Bulgarien) (Barslund et al. 2017, S. 1-2). Während Entsendungen keineswegs nur zwischen Niedrig- und Hochlohnländern stattfinden, ist dieses Segment in den letzten Jahren am stärksten gewachsen (Pacolet und De Wispelaere 2018).

Abb. 1 illustriert das Einkommensgefälle in der europäischen Bauwirtschaft, auf die knapp 50\% aller Entsendungen in der EU entfallen. Wenn wir die für Arbeitgeber entstehenden gesamten Arbeitskosten betrachten, wird erkennbar, dass nicht nur die Lohnkosten stark divergieren, sondern auch die entsprechenden Sozialabgaben und Steuern. Mit Ausnahme von Schweden sind es vor allem die kontinentaleuropäischen „Bismark'schen“ Wohlfahrtsstaaten (Belgien, Deutschland, Frankreich, Niederlande, Österreich), die nicht nur vergleichsweise hohe Löhne haben, sondern auch hohe Sozialabgaben. Somit besteht insbesondere hier für Firmen eine ,spezifische Anreizstruktur" (Staples et al. 2013), auf grenzüberschreitende Entsendearbeit zuzugreifen, um Arbeitskosten zu senken und einen Bedarf an „flexiblen“ Arbeitskräften abzudecken. Es ist daher vermutlich kein Zufall, dass kontinentaleuropäische Wohlfahrtstaaten die wichtigsten Zielländer für Entsendungen in der europäischen Bauwirtschaft sind (vgl. Abb. 2). In dieser Hinsicht steht die verstärkte Nutzung von Dienstleistungsmobilität nicht nur für eine Flexibilisierung der Arbeitsbeziehungen, sondern auch für ein ,Regimeshopping“ (Dølvik et al. 2014) zwischen unterschiedlichen sozialen Sicherungssystemen in Europa.

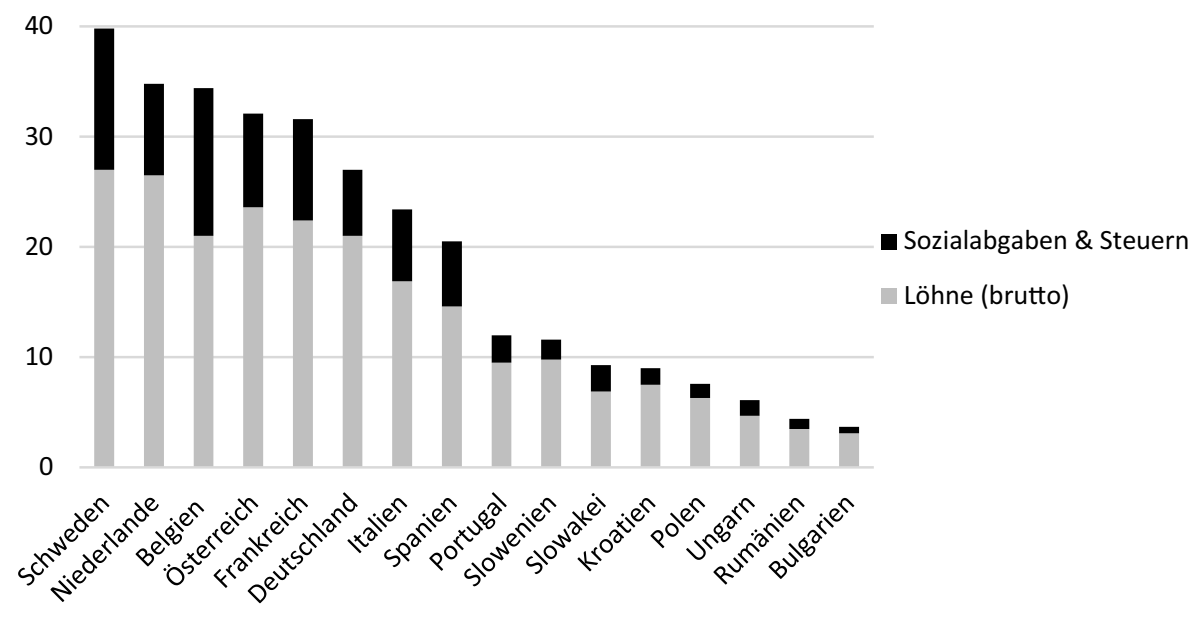

Abb. 1 Arbeitskosten in der europäischen Bauwirtschaft 2016 (pro Stunde, in Euro). Quelle: Eurostat (2017) 


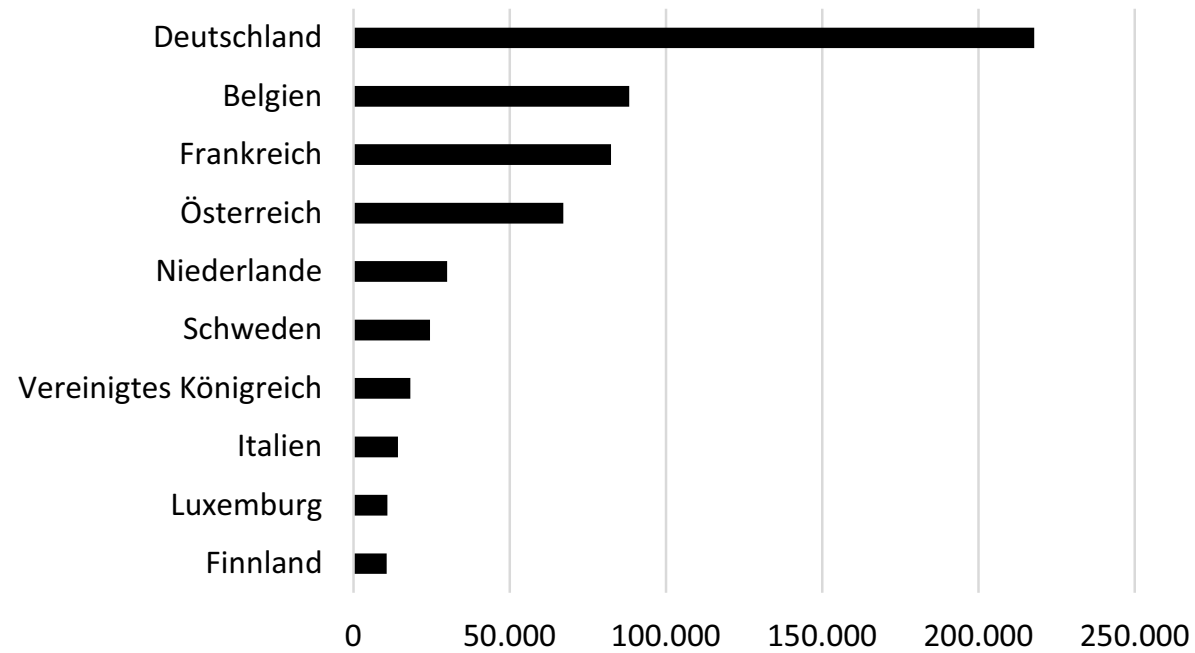

Abb. 2 Entsendungen in der europäischen Bauwirtschaft (10 wichtigste Zielländer) (2016). Quelle: Pacolet und De Wispelaere (2018, S. 26, 29) (eigene Berechnungen)

\section{Methodisches Vorgehen}

Um zu untersuchen, welche Herausforderungen mit einem Anstieg von Entsendungen in Österreich verbunden sind, wurden acht ExpertInneninterviews mit Arbeitgeber- und ArbeitnehmerInnenvertretungen sowie mit VertreterInnen (para-)staatlicher Behörden geführt (s. Anhang). Qualitative ExpertInneninterviews sind eine probate Forschungsmethode, um das spezifische Fachwissen von Personen zu erschließen, welches diese aufgrund ihrer beruflichen Position besitzen (Bogner et al. 2005). Solche Interviews passen vor allem dann, wenn das Themengebiet, wie im Fall von Entsendearbeit, noch relativ unerforscht ist und es sich um eine explorative Studie handelt. Zudem bieten sie sich an, wenn sich der Feldzugang schwierig gestaltet. Dies ist bei Entsendearbeit aufgrund von Sprachbarrieren, relativ kurzen Arbeitseinsätzen und räumlicher Segregation der Fall (Staples et al. 2013, S. 273-274).

Um eine Vergleichbarkeit der Interviewdaten zu gewährleisten, wurden systematisierende ExpertInneninterviews durchgeführt, welche sich vor allem in der Industriesoziologie einer relativ großen Beliebtheit erfreuen. Bei diesem Interviewtyp wird die Vergleichbarkeit der Interviewdaten durch einen relativ stark ausdifferenzierten Leitfaden ermöglicht (Bogner et al. 2005, S. 38). Dementsprechend wurden die Leitfäden nach den folgenden Themen strukturiert: die Entwicklung von Entsendearbeit seit 2011, die damit verbundenen Herausforderungen für den Arbeitsmarkt, nationale und supranationale Regulierungsmaßnahmen sowie die Rolle der Sozialpartnerschaft.

Die Interviews, die unter inhaltsanalytischen Gesichtspunkten mithilfe des Softwareprogramms MAXQDA ausgewertet wurden, wurden noch mit anderen Quellen (Policy-Dokumente, Internetseiten und Zeitungsartikel) trianguliert (Gläser und Laudel 2009). Zudem wurden öffentlich zugängliche Arbeitsmarktdaten in die Analyse mit einbezogen, ebenso wie Daten zu Herkunft und Zusammensetzung von Entsen- 
debetrieben und Beschäftigten, die dem Autor von der Bauarbeiter-Urlaubs- und Abfertigungskasse (BUAK) zur Verfügung gestellt wurden.

\section{Der österreichische Arbeitsmarkt nach der Öffnung: Eine Präferenz für Entsendearbeit?}

Seit der Arbeitsmarktöffnung 2011 ist ein starker Anstieg von Entsendungen nach Österreich zu beobachten. Wurden 2010 noch knapp 60.000 Entsendungen (A1-Sozialversicherungsformulare) registriert, waren es 2016 schon über 120.000 Entsendungen. ${ }^{5}$ Diese Entwicklung ging nahezu ausschließlich auf Entsendungen aus den neuen Mitgliedsstaaten zurück, deren Zahl sich im gleichen Zeitraum von knapp 17.000 Entsendungen auf über 78.000 mehr als vervierfacht hat, der höchste Anstieg in der erweiterten EU (Europäische Kommission 2012, S. 13; Pacolet und De Wispelaere 2018, S. 21). Die hauptsächlichen Herkunftsländer waren Slowenien, Ungarn und die Slowakei, und die hauptsächlichen Zieldestinationen waren vor allem grenznahe Regionen im Burgenland, in der Steiermark, in Niederösterreich, in Kärnten, im Wiener Umland sowie in Oberösterreich (Mühlviertel) (Schmatz und Wetzel 2014, S. 28). Somit ist geografische Nähe ein wichtiger Prädiktor für Entsendungen. In dieser Hinsicht ist Österreich in einer außergewöhnlichen Lage, da es als einziges Land der alten Mitgliedsstaaten an vier der neuen Mitgliedsstaaten grenzt (Slowakei, Slowenien, Tschechien und Ungarn), die zudem alle über ein deutlich niedrigeres Lohnniveau verfügen.

Dienstleistungsmobilität findet vor allem im Baubereich statt, der seit der Öffnung an Bedeutung gewonnen hat und auf den mittlerweile knapp 60\% aller Entsendungen nach Österreich entfallen. Innerhalb der Branche verteilen sich Entsendungen in einem etwa gleichen Ausmaß auf das Bauhauptgewerbe (Hoch- und Tiefbau) und das Baunebengewerbe (z.B. Installation oder Malerei) (Schmatz und Wetzel 2014, S. 23). Wenn die nationale Zusammensetzung der Entsendebetriebe betrachtet wird, fällt auf, dass sich diese grundlegend verändert hat. Während im Jahr vor der Arbeitsmarktöffnung 90\% aller Betriebe aus der ,,alten“ EU kamen, vor allem aus Deutschland, kommen mittlerweile über $70 \%$ aus den NMS (Schmatz und Wetzel 2014, S. 24). Dies deutet darauf hin, dass grenzüberschreitende Entsendearbeit zunehmend von einer Logik des ,kompetitiven Subcontracting“ (Harvey 2003) dominiert ist.

Der hauptsächliche „Wettbewerbsvorteil“" von Entsendebetrieben aus den NMS sind ihre niedrigeren Arbeitskosten. Zwar gilt seit dem Arbeitsvertragsrecht-Anpassungsgesetz (AVRAG) von 1993 das kollektivvertragliche Entgelt auch für entsandte

\footnotetext{
5 A1-Sozialversicherungsformulare (früher E101) werden vom jeweiligen Versicherungsträger im Herkunftsland (z. B. von der Wiener Gebietskrankenkasse) für die entsandte Person ausgestellt und geben unter anderem den Zielort der Entsendung an. Diese Bescheinigungen geben nicht unbedingt Auskunft über die Gesamtzahl der entsandten Personen, da für eine Person im Fall einer wiederholten Entsendung auch mehrere A1-Formulare ausgestellt werden können. Gleichzeitig ist davon auszugehen, dass diese Bescheinigungen das Ausmaß von grenzüberschreitender Mobilität unterschätzen, da vor allem bei kurzfristigen Entsendungen Firmen gelegentlich darauf bedacht sind, das administrative Meldeverfahren zu umgehen (Riesenfelder et al. 2012, S. 281).
} 
Beschäftigte, aber Erfahrungen aus der Bauwirtschaft legen nahe, dass die entsprechenden Löhne oftmals nicht gezahlt werden. Kontrollen der BUAK haben ergeben, dass bei $40 \%$ aller kontrollierten ausländischen Baufirmen ein Verdacht auf kollektivvertragliche Unterentlohnung vorlag. Im Vergleich dazu war dies bei nur einem Prozent der inländischen Baufirmen der Fall (Die Presse 2017). Selbst wenn inländische Kollektivvertragslöhne gezahlt werden, können ausländische Entsendebetriebe ihre Dienstleistungen immer noch günstiger anbieten, da bei Entsendungen in der Regel nicht die relativ hohen Sozialversicherungsbeiträge des österreichischen Ziellandes anfallen, sondern die deutlich geringeren Beiträge des Herkunftslandes (vgl. Abb. 1).

Entsendearbeit illustriert somit die Herausforderungen, die mit einem transnationalen Mobilitätsraum verbunden sind, der von erheblichen Einkommensunterschieden geprägt ist. Während der durchschnittliche Bruttostundenlohn im Bausektor in Österreich 2016 bei knapp $24 €$ lag, lag er bei den wichtigsten Entsendeländern Slowenien $(9,80 €)$, Slowakei $(6,90 €)$ und Ungarn $(4,70 €)$ deutlich darunter (Eurostat 2017). ${ }^{6}$ Diese erheblichen Unterschiede können für entsandte Beschäftigte einen Anreiz darstellen, für einen Lohn zu arbeiten, der unterhalb der kollektivvertraglichen Mindestlöhne liegt, aber immer noch deutlich besser ist als im Herkunftsland. Mit anderen Worten, ein „schlechter“ Lohn in Österreich kann ein ,guter“ Lohn in Ungarn oder der Slowakei sein, vor allem dann, wenn der Lebensmittelpunkt im Herkunftsland bleibt (vgl. auch Wiesböck 2016).

Grundsätzlich stellt die Beschäftigung von ausländischen MitarbeiterInnen keine neue Entwicklung in der Baubranche dar, die schon immer aufgrund ihres saisonalen Charakters und des hohen Anteils an arbeitsintensiven, geringer qualifizierten Tätigkeiten einen Pull-Faktor für MigrantInnen darstellte (Balch et al. 2004). Neu ist allerdings der Stellenwert, den Entsendearbeit gegenüber individueller Arbeitsmigration einnimmt. Während nach Daten der BUAK die Zahl der ausländischen Beschäftigten, die bei einer österreichischen Baufirma direkt angestellt sind, zwischen 2011 und 2016 um knapp 20\% gestiegen ist, ist im gleichen Zeitraum die Zahl der Entsendungen um über hundert Prozent gestiegen (BUAK 2017a, 2017b). Dies deutet darauf hin, dass Firmen in einigen Bereichen zunehmend eine Präferenz für ausländische Entsendearbeit entwickeln, um ihren Bedarf an „flexiblen“ Arbeitskräften zu decken und Kosten zu senken.

Wie hat sich der verstärkte Rekurs auf Entsendearbeit auf den österreichischen Arbeitsmarkt ausgewirkt? Während bereits erste Studien zu den Auswirkungen eines Anstiegs von Ost-West-Migration vorliegen (z. B. Huber und Böhs 2012), gibt es noch keine vergleichbaren Untersuchungen zu den Arbeitsmarkteffekten von Dienstleistungsmobilität. Studien aus Deutschland legen nahe, dass ein Anstieg von Entsendearbeit in einzelnen Branchen zu Verdrängungseffekten geführt hat (Bosch 2013; Wagner und Hassel 2015). Erste Indizien könnten auf eine ähnliche Entwicklung in Österreich hindeuten. So ist beispielsweise seit der Arbeitsmarktöffnung die Arbeitslosenquote von türkischstämmigen Bauarbeitern überdurchschnittlich stark angestiegen (Sozialministerium 2018). Dies scheint die These zu bestätigen, dass vor allem längerfristig ansässige ausländische Arbeitskräfte stärker von der neuen Ar-

\footnotetext{
${ }^{6}$ Beim Vergleich der Löhne handelt es sich um direkte Wechselkurse.
} 
beitsmobilität aus den NMS betroffen sind (Huber und Böhs 2012, S. 41). Auch eine Analyse von Daten des Mikrozensus deutet darauf hin, dass es in grenznahen Regionen im Burgenland, der Steiermark, in Niederösterreich und im Wiener Umland, die einen starken Anstieg von Entsendungen im Baubereich erfahren haben, zu Verdrängungseffekten im unteren Qualifikationssegment gekommen ist (Schweighofer 2012, S. 612).

Unstrittig ist, dass Entsendungen zu Kontroversen über Lohndumping und die Einhaltung von Arbeitsstandards geführt haben. Üblicherweise wird von Lohn- und Sozialdumping gesprochen, wenn ,foreign or local companies employ their workers at conditions inferior to those laid down in the host country's employment regulations or collective agreements“ (Bernaciak 2012, S. 26). Inwieweit ist dieses Phänomen in der österreichischen Baubranche zu beobachten? Dies soll nun näher mithilfe der ExpertInneninterviews ergründet werden.

\section{5 „Varianten des Lohndumpings“ in der Baubranche}

Die ExpertInneninterviews legen nahe, dass Arbeitsmarktakteure im Baubereich mit verschiedenen „Varianten des Lohn- und Sozialdumpings“ konfrontiert sind. Die Umgehung von Arbeits- und Sozialstandards stellt nicht unbedingt ein neues Phänomen in einer Branche dar, die aufgrund von Subunternehmerketten, Scheinselbstständigkeit und einer vergleichsweise hohen Fluktuation von Arbeitskräften relativ anfällig für irreguläre Praktiken ist (Balch et al. 2004). ${ }^{7}$ Oder in den Worten einer Vertreterin der BUAK: ,Wir haben eine gewisse kriminelle Energie in der Bauwirtschaft, die hat es immer schon gegeben, und mit der werden wir in der einen oder anderen Form immer konfrontiert sein." Es besteht aber weitgehend Einigkeit unter den befragten ExpertInnen, dass die Arbeitsmarktöffnung zu einer qualitativ neuen Situation geführt hat:

[D]ie teilweise bestehende Erwartung [...], dass nach der Arbeitsmarktöffnung eine Zunahme [von Entsendungen] erfolgt, aber dann nach zwei, drei Jahren die Zunahme gleich bleiben wird, das ist nicht eingetreten [...] es ist weiterhin eine starke Zunahme [...] das ist vor allem natürlich der Baubereich, aber auch andere Bereiche, wo es vor allem um gering qualifizierte Personen geht [...] Es geht im Endeffekt darum, durch Bezahlung von Löhnen, die unter dem österreichischen Kollektivvertrag liegen, sich hier billiger anbieten zu können (Interview, AK Wien, Abtl. Sozialpolitik).

(W)ir merken, es springt an. Die meisten Branchen, in die entsandt wird, das sind Gewerbe, Baubranchen und Dienstleistungen[...] die Befürchtung bei einigen Branchen, dass es zu einer Wettbewerbsverzerrung kommt (Interview, WKO, Abtl. Sozialpolitik).

\footnotetext{
7 Eine nichtrepräsentative Auswertung von Daten der BUAK fand im Jahr 2012, dass bei knapp $70 \%$ der kontrollierten Beschäftigten, die einer vermeintlich selbstständigen Tätigkeit im Baubereich nachgingen, ein Verdacht auf Scheinselbstständigkeit vorlag (Schmatz und Wetzel 2014, S. 58).
} 
„Varianten des Lohndumpings“ können in einem Kontinuum von regulärer und irregulärer Beschäftigung angeordnet werden. Wie schon erwähnt, wird üblicherweise von Lohn- und Sozialdumping gesprochen, wenn Firmen Arbeitskräfte zu ungünstigeren Bedingungen beschäftigen, als in den Kollektivverträgen oder Verordnungen am Arbeitsort festgelegt ist. Allerdings legen Erfahrungen aus der Baubranche nahe, dass Lohndumping nicht notwendigerweise die Unterschreitung von Kollektivverträgen voraussetzt, wenn die bisherige Norm eine Überzahlung war:

[I]m Tunnelbau werden Mineure aus den neuen Mitgliedsstaaten [eingesetzt]. Dort ist es aber ein legales Lohndumping [...] Ein Mineur geht normalerweise nicht für den kollektivvertraglichen Lohn untertags arbeiten, da waren Überzahlungen von bis zu $30 \%$ üblich. Und die Mineure aus den neuen Mitgliedsstaaten, die gehen zum Kollektivvertragslohn auch hinunter, weil es immer noch deutlich mehr ist, als sie zu Hause verdienen würden. Deswegen sage ich, dies ist legales Lohndumping. Das ärgert natürlich die österreichische Gewerkschaft und ihre Mitglieder, aber das ist nicht ungesetzlich (Interview, WKO Geschäftsstelle Bau).

Ein Beispiel für Lohndumping, welches nicht mehr als „legal“ bezeichnet werden kann, ist die inkorrekte kollektivvertragliche Einstufung von entsandten Beschäftigten. In diesem Zusammenhang werden immer wieder Fälle von Bauarbeitern bekannt, die trotz fachlicher Qualifikation als Hilfsarbeiter eingestuft werden (Riesenfelder et al. 2012, S. 376-378). Solch eine Form von kollektivvertraglicher Unterentlohnung ist aber keineswegs auf Entsendearbeit beschränkt, wie eine Vertreterin der BUAK betont:

[D]a geht es dann oft darum, ist das ein Hilfsarbeiter, ist das ein angelernter Arbeiter, da geht es um Tricks, wie halt die Arbeitgeberseite versucht, die Löhne, das Lohnniveau zu drücken[...] aber auch da ist eben ein Kontrolleur gefordert, Feststellungen zu treffen, die eindeutig belegen, dass das jetzt kein Hilfsarbeiter ist [...] Aber das ist auch nicht nur ein Entsendethema, dieses Thema kennen wir seit Jahren und damit haben wir immer zu tun (Interview, BUAK (1)).

Auch ,Scheinentsendungen“ bewegen sich in einer Grauzone von regulärer und irregulärer Beschäftigung. Dabei werden Arbeitskräfte gezielt im Herkunftsland (oder vereinzelt sogar im Zielland) für eine Entsendung angeworben, nicht selten von einer „Briefkastenfirma“, die keiner eigenständigen wirtschaftlichen Tätigkeit im Ausland nachgeht. Zwar werden die Arbeitskräfte meistens bei der entsprechenden Sozialversicherung im Herkunftsland angemeldet, um eine „,reguläre“ Entsendung zu dokumentieren, die entsprechenden Sozialbeiträge werden aber nicht unbedingt gezahlt. Auch sind Scheinentsendungen immer wieder von Formen kollektivvertraglicher Unterentlohnung begleitet, wie Beispiele aus der Bauwirtschaft dokumentieren (Riesenfelder et al. 2012, S. 391-393).

Bei Verstößen gegen das kollektivvertragliche Mindestentgelt sind Kontrollbehörden und andere Arbeitsmarktakteure mit verschiedenen Dumpingpraktiken konfrontiert. So wurden Fälle bekannt, in denen entsandte Beschäftigte den kollektivvertraglichen Mindestlohn ausgezahlt bekamen, aber einen Teil des Lohnes wieder an den Arbeitgeber im Heimatland abführen mussten (Schmatz und Wetzel 2014, 
S. 44). In anderen Fällen liegen die ausgezahlten Löhne „nur“ geringfügig unter den entsprechenden Kollektivlöhnen. Bisweilen kommen aber auch eklatante Formen der Unterzahlung ans Tageslicht:

[B] ei den Unterentlohnungen bei entsandten Arbeitnehmern [bekommen wir] mit Befragungen durchaus Informationen [...] er sagt, er verdient, er kriegt vier Euro. Eigentlich sollten es 13 sein, solche Fälle gibt es (BUAK (2)).

Dumpingpraktiken sind vor allem im Rahmen längerer Subunternehmerketten zu beobachten. Durch die (wiederholte) Vergabe von Teilen des Bauauftrages entstehen nicht nur ,,intransparente Marktbeziehungen“ (Hofstadler et al. 2016, S. 26), sondern es wird auch ein Preisdruck erzeugt, der vor allem über niedrige Arbeitskosten ausgetragen wird. Dabei sind grenzüberschreitende Vergabeketten meistens von einer hierarchischen Struktur zwischen inländischen und ausländischen Firmen gekennzeichnet:

[W]enn jetzt die öffentliche Hand etwas baut, ist da der Auftragnehmer, der Generalunternehmer [...] ein österreichischer Betrieb. Das ist ja erst in der Subkette, wo die ausländischen Betriebe dann herangezogen werden, das ist so im Allgemeinen unsere Erfahrung. [...] [E]igentlich gibt es immer eine österreichische Baufirma, die dann einen Teil des Auftrages an eine Subfirma billiger vergibt (Interview BUAK (2)).

Durch die Subvergabe von einzelnen Bauleistungen kann die österreichische Auftragsfirma nicht nur den Kostendruck nach ,unten“ weitergeben, sondern auch Verantwortung für die Einhaltung von Standards an Subfirmen externalisieren, eine Praxis, die auch in anderen Branchen zu beobachten ist (Flecker 2009).

Im Baubereich konnten somit verschiedene „Varianten des Lohndumpings“ identifiziert werden, die von der Unterschreitung bisheriger Normen der Überzahlung über die falsche kollektivvertragliche Einstufung und „Scheinentsendungen“ bis hin zu eklatanten Formen der Unterzahlung reichen. Dabei erweisen sich vor allem längere Subunternehmerketten als anfällig für Dumpingpraktiken, da sie oftmals von einem informellen Arbeitsumfeld geprägt sind, in dem entsprechende Mindeststandards kaum eingehalten werden.

\section{Das Lohn- und Sozialdumping-Bekämpfungsgesetz: Ein „treffsicheres“ Kontrollregime?}

Um irreguläre Praktiken im Zusammenhang mit grenzüberschreitender Mobilität zu unterbinden, wurde 2011 das Lohn- und Sozialdumping-Bekämpfungsgesetz (LSDBG) verabschiedet. Vor allem der Österreichische Gewerkschaftsbund (ÖGB) und die Bundesarbeitskammer (BAK) hatten sich im Hinblick auf die Arbeitsmarktöffnung für neue gesetzliche Maßnahmen ausgesprochen. Nach anfänglichem Zögern stimmte auch die Wirtschaftskammer Österreich (WKO) dem neuen Gesetz zu, nicht 
zuletzt, weil ihre einflussreiche Sparte „Gewerbe und Handwerk“ einen Unterbietungswettbewerb durch ausländische Dienstleistungsfirmen befürchtete. ${ }^{8}$

Im LSD-BG heißt es, dass entsandte Beschäftigte „für die Dauer der Entsendung zwingend Anspruch auf zumindest jenes gesetzliche, durch Verordnung festgelegte oder kollektivvertragliche Entgelt [haben] [...], das am Arbeitsort vergleichbaren Arbeitnehmern von vergleichbaren Arbeitgebern gebührt“ (§3, Abs. 3, LSD-BG). Dies schließt auch Sonderzahlungen und Zuschläge mit ein. Somit hat Österreich eines der umfassendsten Regulierungsregime in Europa, welches, wie auch schon im früheren AVRAG, das Prinzip von ,gleicher Lohn für gleiche Arbeit“ weitgehend durchsetzt (Krings 2016). ${ }^{9}$

Ein wesentlicher Bestandteil des neuen Gesetzes ist die Durchführung von Lohnkontrollen durch die Finanzpolizei und im Fall der Bauwirtschaft durch die BUAK. Letztere sehen die Arbeitsmarktöffnung nicht nur als eine Herausforderung, sondern auch als eine Chance, um für ,bessere Verhältnisse“ in der Branche zu sorgen. Dadurch, dass das Thema Entsendungen eine stärkere Präsenz in der öffentlichen Debatte erfahren hat, ist der Spielraum für neue Kontrollmaßnahmen größer geworden:

[O]hne diese Arbeitsmarktöffnung, wie wir sie 2011 erlebt haben, wären wir in diesen verschiedenen Bereichen [...] nie so weit gekommen [...] Früher haben wir nie dieses Einverständnis der Sozialpartner gehabt, dass Löhne kontrolliert werden, dass die BUAK und andere Institutionen auf den Baustellen kontrollieren, dass wir mit den Arbeitnehmern sprechen, dass wir von den Baubetrieben alle Unterlagen über Auftragsverteilungen bekommen, dass wir eine Baustellendatenbank haben, wo die Baustellen dokumentiert werden [...] es eröffnet uns [...] die Chance, für wesentlich bessere Verhältnisse in der Branche zu sorgen, weil jetzt auch das politische Interesse wieder vorhanden ist, etwas zu tun (Interview BUAK (2)).

Die zentrale Rolle (para-)staatlicher Akteure bei der Durchführung von Lohnkontrollen muss vor dem Hintergrund des Rückgangs der gewerkschaftlichen Organisierung und einer wachsenden ,,betrieblichen Vertretungslücke“ (Hermann und Flecker 2006) betrachtet werden. Anders als beispielsweise in Schweden sind ArbeitnehmerInnenvertretungen in Österreich weniger in der Lage, über die Einhaltung von Arbeitsstandards in den Betrieben oder auf Baustellen zu wachen. Dabei zeigt sich ein Durchsetzungsproblem vor allem in kleineren in- und ausländischen Betrieben, die meistens über keine betriebliche Interessenvertretung verfügen (Riesenfelder et al. 2012, S. 405-406). Angesichts begrenzter eigener Ressourcen und der Schwie-

\footnotetext{
8 Ein weiterer Faktor war, dass das LSD-BG Teil eines sozialpartnerschaftlichen Interessenausgleichs war, in dem der ÖGB und die BAK im Gegenzug der Einführung einer Rot-Weiß-Rot-Karte für hochqualifizierte MigrantInnnen zustimmten, ein Kernanliegen der Arbeitgeberseite (Krings 2013).

9 Das LSD-BG war ursprünglich im AVRAG verankert, wurde aber im Rahmen einer Novelle, die auch der Implementierung der EU-Durchsetzungsrichtlinie 2014/67/EU diente, 2017 in ein eigenes Gesetz überlassen. Neben Maßnahmen zur besseren Vollstreckung von Verwaltungsstrafen im Ausland beinhaltet das neue Gesetz auch die Schaffung einer Auftraggeberhaftung für die Lohnansprüche entsandter Beschäftigter im Baubereich.
} 
rigkeit, die Entgeltansprüche von entsandten Beschäftigten durchzusetzen, wird von der ArbeitnehmerInnenseite die Wichtigkeit von Kontrollen betont:

Die betroffenen Arbeitnehmer klagen ihre Ansprüche nicht ein, aus verschiedenen Gründen, und selbst wenn sie sie einklagen würden, das Risiko für den Arbeitgeber wäre denkbar gering, denn schlimmstenfalls müsste er auch nur das zahlen, was er sowieso zahlen müsste. Insofern war auch schon seit vielen Jahren klar, dass es [...] auch notwendig ist, hier bestimmte Kontrollen und eben Verwaltungsstrafen vorzusehen (Interview, AK Wien, Abtl. Sozialpolitik).

Ursprünglich kursierte unter den Sozialpartnern ein Entwurf, der Verwaltungsstrafen nur für ausländische Dienstleistungserbringer vorsah. Dieser Entwurf war aber nicht konform mit geltendem Europarecht und musste nachgebessert werden, sodass die neuen Bestimmungen auch für inländische Unternehmen zu gelten haben. Dieser Tatbestand sorgte für wenig Begeisterung auf der Arbeitgeberseite (Interview, BMASK (1)). Tatsächlich befand sich die WKO bei den Verhandlungen zum LSDBG in einem gewissen Dilemma. Man wollte zwar einen Unterbietungswettbewerb durch ausländische Dienstleister vermeiden, gleichzeitig wollte man aber keine bürokratische Mehrbelastung für österreichische Betriebe schaffen. Dieses Dilemma hat sich seit Inkrafttreten des neuen Gesetzes eher noch verschärft:

[E]s gibt Branchen [...] z. B. die Baubranche, vor allem in den Grenzregionen, Burgenland und Umgebung, die sehr froh sind, dass endlich kontrolliert wird, dass die Strafen hoch sind und abschreckend sind. Und es gibt wiederum andere Branchen, die sagen, eine fehlerhafte Lohnverrechnung, oder nach Ansicht der Behörde in die falsche kollektivvertragliche Entlohnungsgruppe [eingestuft] und jetzt muss ich schon $50.000 €$ Strafen zahlen (Interview, WKO, Abtl. Sozialpolitik). ${ }^{10}$

Insgesamt hat sich auf der Arbeitgeberseite eine Wahrnehmung durchgesetzt, dass das LSD-BG zu wenig ,treffsicher“" ist und die „Falschen“ (aka österreichische Betriebe) trifft:

Es [das LSD-BG] ist zwar jetzt schon fast sechs Jahre in Geltung [...] aber noch immer funktioniert es nicht und ist nicht so treffsicher, wie man sich das damals gewünscht hat [...] die große Schwäche der derzeitigen Rechtslage [ist], dass eben inländische Betriebe genauso hoch gestraft werden und die Strafen auch vollstreckt werden, und die ausländischen Betriebe verlassen sich darauf, dass ihre [...] Regierungen [...] die Strafen nicht so konsequent eintreiben (Interview, WKO, Abtl. Sozialpolitik).

Diese Sichtweise wird von der Industriellenvereinigung (IV) geteilt, die dem LSD-BG von Anfang an eher kritisch gegenüberstand. Die IV, die nicht Teil der offiziellen Sozialpartnerschaft ist, vertritt vor allem die Interessen größerer Industrieunternehmen, für die es beim Thema Entsendungen zuvorderst um konzerninterne Mobilität geht und weniger um die Sorge vor einem Unterbietungswettbewerb durch

$1050.000 €$ ist die im LSD-BG festgesetzte Höchststrafe im Wiederholungsfall für Unterentlohnung oder das Nichtbereithalten von Lohnunterlagen. 
ausländische Dienstleister. ${ }^{11}$ Die IV bemängelt zu viel „Bürokratie“ durch das LSDBG und fordert, das neue Gesetz auf ,echte Fälle“ von Lohn- und Sozialdumping zu beschränken:

[M]it dem Lohn- und Sozialdumping-Bekämpfungsgesetz [ist] in Österreich ein hoher Administrationsaufwand für die Betriebe bezüglich Meldungen, Dokumentation, Vorlagepflicht usw. verbunden [...] [A] us unserer Sicht ist es wesentlich und wichtig, dass man die Normen halt so gestaltet, dass man sie auf echte Fälle von Lohn- und Sozialdumping fokussiert [...] Fälle, wo es um ich sage einmal kleinere Fehler in der Lohnverrechnung geht, um schwierige Einstufungsfragen in der Auslegung von komplexen Kollektivvertragsregelungen, solche, ja, Abwägungsfragen, Beurteilungsfragen, sind aus unserer Sicht kein Lohn- und Sozialdumping (Interview, Industriellenvereinigung).

Solche Aussagen legen nahe, dass es in der Debatte um Entsendearbeit auch um eine Deutungshoheit von Lohndumping geht. Wird jedenfalls die oben zitierte Definition zugrunde gelegt, dann konstituieren auch geringe Abweichungen von Kollektivverträgen eine Form von Lohndumping, auch wenn sie möglicherweise unbeabsichtigt erfolgen.

Weitgehend Einigkeit besteht unter den befragten ExpertInnen, dass es Defizite bei der grenzüberschreitenden Vollstreckung von Verwaltungsstrafen gibt. In Österreich hat sich die Zahl der Anzeigen wegen kollektivvertraglicher Unterentlohnung in den letzten Jahren erhöht. Dies dürfte nicht nur auf einen Anstieg der Entsendungen zurückzuführen sein, sondern auch auf einen Anstieg der Kontrollen (Szigetvari 2018a). Allerdings endet die Strafverfolgung meistens an der nationalen Grenze, wie ein Vertreter der BUAK bemerkt:

Wir haben die Anzeige zu erstatten, wir sind im Verwaltungsverfahren Partei und es kommt dann auch zur Bestrafung [...] und die Verfahren, die finden alle statt, aber dass das Geld dann wirklich bei demjenigen, der es zahlen sollte, eingehoben wird, findet eher nicht statt (Interview, BUAK (2)).

Bei diesem Tatbestand scheint es sich um ein strukturelles Problem in der EU zu handeln. Zwar wurde auf Grundlage der vier Freiheiten ein transnationaler Wirtschafts- und Mobilitätsraum geschaffen, gleichzeitig wurde aber verabsäumt, entsprechende Kontroll- und Verfolgungsinstanzen zu etablieren (Bosch 2013, S. 283). Durch die EU-Durchsetzungsrichtlinie 2014/67/EU soll zwar die grenzüberschreitende Kooperation zwischen den Behörden im Rahmen des Internal Market Information System verbessert werden, aber noch ,steckt die Zusammenarbeit der Behörden [...] in den Kinderschuhen“ (Gagawczuk 2018, S. 2). Einmal mehr zeigt sich, dass es ungleich schwerer fällt, regulative Instanzen im Sinne einer „positiven Integration“ auf supranationaler Ebene zu schaffen.

\footnotetext{
11 Nicht zuletzt aufgrund der Lobbyarbeit von IndustrievertreterInnen enthält das neue LSD-BG von 2017
} Ausnahmeregelungen für konzerninterne Entsendungen. 


\section{Schlussfolgerungen}

Dieser Beitrag hat den Anstieg und die Regulierung von Entsendearbeit am Beispiel der österreichischen Bauwirtschaft untersucht. Die hauptsächliche Dynamik für den starken Anstieg von Entsendungen war die Öffnung des Arbeitsmarktes 2011 im Zusammenspiel mit Institutionen, Ungleichheit und Geografie. Vor allem in kontinentaleuropäischen Wohlfahrtsstaaten besteht für Firmen ein Anreiz, auf Dienstleistungsmobilität zuzugreifen, um vergleichsweise hohe Lohnnebenkosten zu umgehen und mehr „Flexibilität“ in den Arbeitsbeziehungen zu erreichen (Hunger 2000). Aus dieser Perspektive betrachtet ist die verstärkte Nutzung von Entsendearbeit nachfrageorientiert. Sie ist aber auch angebotsorientiert, da sich Unternehmen aus den NMS in anderen Arbeitsmärkten frei anbieten können, wobei ihr „Wettbewerbsvorteil“" niedrigere Arbeitskosten sind. Somit zeigt das österreichische Beispiel, dass sich Angebot und Nachfrage in einem transnationalen Mobilitätsraum, der von erheblichen Einkommensungleichheiten gekennzeichnet ist, in einem dynamischen Verhältnis zueinander entwickeln können.

Die Befunde dieses Beitrags legen nahe, dass im Baubereich die grenzüberschreitende Erbringung von Dienstleistungen zunehmend von einer Logik des „kompetitiven Subcontracting“ (Harvey 2003) dominiert ist. In solch einem wirtschaftlichen Umfeld, in dem der Wettbewerb vor allem über niedrige Kosten ausgetragen wird, scheinen Dumpingpraktiken relativ weit verbreitet zu sein. In diesem Beitrag konnten verschiedene „Varianten des Lohndumpings“ identifiziert werden, die in einem Kontinuum von regulärer und irregulärer Beschäftigung stehen. Inwieweit diese Entsendearbeit zu Verwerfungen am österreichischen Bauarbeitsmarkt geführt hat, konnte nicht abschließend geklärt werden, erste Indizien deuten aber auf einen Verdrängungswettbewerb im unteren Qualifikationssegment hin.

Bis zu einem gewissen Grad sind die Befunde dieses Beitrags spezifisch für den Baubereich, der durch einen hohen Anteil an arbeitsintensiven Tätigkeiten, einen projektförmigen Produktionsprozess und längere Subunternehmerketten geprägt ist. Allerdings ist die kompetitive Auslagerung von Dienstleistungen auch in anderen Branchen wie dem Reinigungsgewerbe oder der Transportwirtschaft zu beobachten, wenn auch nicht in einem vergleichbaren Ausmaß (Haidinger 2016; Schmatz und Wetzel 2014).

In analytischer Hinsicht erscheint es sinnvoll, zwischen Entsendearbeit und individueller Arbeitsmigration zu unterscheiden, nicht zuletzt, weil ihnen eine unterschiedliche „Logik der Integration“ in den Arbeitsmarkt zugrunde liegt. In der sozialen Wirklichkeit grenzüberschreitender Mobilität kann diese Unterscheidung aber nicht immer aufrechterhalten werden. In der EU ist ein transnationaler Markt für Niedriglohnarbeit entstanden, in dem die Grenzen zwischen Entsendearbeit, Scheinselbstständigkeit und abhängiger Beschäftigung oftmals fließend sind (Dølvik et al. 2014, S. 156). Was diese verschiedenen Formen arbeitsbezogener Mobilität gemeinsam haben, sind ein temporärer Charakter, eine geringfügige Bezahlung und ein hohes Maß an Prekarität. In dieser Hinsicht kann ein Anstieg von Entsendearbeit als Teil eines allgemeinen Anstiegs von Ost-West-Mobilität betrachtet werden, der mit einer verstärkten Segmentierungstendenz im österreichischen Arbeitsmarkt einhergeht (Eppel et al. 2017). 
Um grenzüberschreitende Mobilität wieder in nationale Arbeitsbeziehungen ,einzubetten“, wurde mit dem LSD-BG ein relativ umfassendes Regulierungsregime geschaffen. Allerdings legen die Ergebnisse dieses Beitrags nahe, dass dieses Regime sowohl in sozialer als auch in räumlicher Hinsicht an seine Grenzen stößt. Entsandte Beschäftigte sind zwar physisch am Arbeitsort präsent, sie arbeiten aber oftmals in segmentierten Arbeitsplätzen, in denen nur begrenzt ein sozialer Austausch mit inländischen Akteuren zustande kommt (vgl. auch Wagner und Lillie 2014). Zudem stößt dieses Regime auch an seine sprichwörtlichen nationalen Grenzen, da verhängte Verwaltungsstrafen im Fall von Unterzahlung kaum im Ausland vollstreckt werden können.

Während das österreichische Regime hauptsächlich auf eine Kontrollstrategie setzt, legt die internationale Forschung nahe, Arbeitsplatzkontrollen durch Maßnahmen zu ergänzen, die auf die verstärkte Einbindung nichtstaatlicher Akteure abzielen (z. B. Dickens 2012; Weil 2010). Eine wichtige Bedeutung wird den Sozialpartnern zugewiesen, die durch die Aushandlung von Kollektivverträgen zu einer (Selbst-)Regulierung des Arbeitsmarktes beitragen können. Die Befunde dieses Beitrags legen aber nahe, dass die regulative Kapazität von Kollektivverträgen vor allem im Rahmen von Subunternehmerketten begrenzt ist. Daher erscheint es geboten, auf einer höheren Ebene anzusetzen, um das Machtgefälle innerhalb von Vergabeketten im Sinne einer „strategischen Durchsetzung“ (Weil 2010) von Arbeitsstandards zu nutzen.

In der internationalen Forschung wie auch in der Policy-Debatte wird in diesem Zusammenhang immer wieder über die Einführung einer Generalunternehmerhaftung diskutiert. Allerdings deutet momentan wenig darauf hin, dass solch eine Maßnahme unter einer schwarz-blauen Bundesregierung politisch realisierbar ist, zumal auch die WKO sie ablehnt (Szigetvari 2018b). Umso mehr bietet es sich daher an, Kontrollmaßnahmen durch Initiativen zu ergänzen, die verstärkt auf die Inklusion mobiler Arbeitskräfte abzielen. In diesem Zusammenhang sind virtuelle Projekte wie die „Entsendeplattform“, die in sieben verschiedenen Sprachen über österreichisches Arbeitsrecht und Kollektivverträge informiert, hervorzuheben, ebenso wie das Projekt „Faire Arbeit“, welches unter maßgeblicher Initiative der Gewerkschaft BauHolz ein zweisprachiges Beratungsbüro im Grenzgebiet zwischen der Steiermark und Slowenien eingerichtet hat. ${ }^{12}$ Diese Initiativen zeigen, dass zur Durchsetzung nationaler Arbeitsstandards zunehmend Strategien erforderlich sind, die über den einzelnen Nationalstaat hinausgehen.

Zuletzt konnten sich die europäischen Arbeits- und SozialministerInnen mehrheitlich (und gegen den Widerstand von Entsendeländern wie Polen und Ungarn) auf eine Reform der Entsenderichtlinie einigen. Zukünftig sollen Entsendungen auf maximal 18 Monate begrenzt sein und das Prinzip ,,gleicher Lohn für gleiche Arbeit am gleichen Ort" soll vom ersten Tag an gelten. Diese Einigung muss noch in Gemeinschaftsrecht überführt und dann in den einzelnen Mitgliedsstaaten umgesetzt werden. Wiederum zeigt sich, dass bei der Regulierung von transnationaler Mobilität eine Verschränkung der nationalen und der supranationalen Ebene zu beobachten ist.

12 Die Webadressen der Projekte sind www.entsendeplattform.at und www.faire-arbeit.at. 
Bei diesem Beitrag handelte es sich um eine explorative Studie zu Entsendearbeit in Österreich, sodass die Reichweite der Ergebnisse begrenzt ist. Nichtsdestotrotz wurden einige Themen identifiziert, die für die zukünftige Forschung nutzbar gemacht werden können. Von großem Interesse wäre, die Arbeitsteilung zwischen inländischen und ausländischen Firmen innerhalb grenzüberschreitender Vergabeketten zu rekonstruieren, ungeachtet der Schwierigkeiten, die beim Feldzugang zu erwarten wären. Auch könnte ein Fokus auf die Mikrostrategien entsandter Arbeitskräfte interessante Einblicke in die subjektiven Verarbeitungsformen mobiler und oftmals prekärer Beschäftigter liefern. So viel scheint festzustehen: Die Auseinandersetzung um transnationale Entsendearbeit wird auch zukünftig ein relevantes Thema sein, nicht nur für Arbeitsmarktakteure, sondern auch für die sozialwissenschaftliche Arbeits- und Migrationsforschung.

Funding Open access funding provided by Johannes Kepler University Linz.

\section{Anhang}

\section{Liste der durchgeführten Interviews}

1. Arbeiterkammer (AK) Wien, Abtl. Sozialpolitik, Wien, 08.03.2017.

2. Bauarbeiter-Urlaubs- und Abfertigungskasse (BUAK), Wien, 10.03.2017 (zwei Personen).

3. Bundesministerium für Arbeit, Soziales und Konsumentenschutz (BMASK), Sektion VII Arbeitsrecht und Zentral-Arbeitsinspektorat, Wien, 09.03.2017 (Interview 1).

4. Bundesministerium für Arbeit, Soziales und Konsumentenschutz (BMASK), Sektion VI Arbeitsmarkt, 29.03.2017 (Interview 2).

5. Industriellenvereinigung (IV), Bereich Arbeit und Soziales, 10.03.2017 (zwei Personen).

6. Österreichischer Gewerkschaftsbund (ÖGB), Referat Rechts- und Kollektivvertragspolitik, 09.03.2017.

7. Wirtschaftskammer Österreich (WKO), Geschäftsstelle Bau, 08.03.2017.

8. Wirtschaftskammer Österreich (WKO), Abtl. Sozialpolitik und Gesundheit, 09.03.2017.

Open Access Dieser Artikel wird unter der Creative Commons Namensnennung 4.0 International Lizenz (http://creativecommons.org/licenses/by/4.0/deed.de) veröffentlicht, welche die Nutzung, Vervielfältigung, Bearbeitung, Verbreitung und Wiedergabe in jeglichem Medium und Format erlaubt, sofern Sie den/die ursprünglichen Autor(en) und die Quelle ordnungsgemäß nennen, einen Link zur Creative Commons Lizenz beifügen und angeben, ob Änderungen vorgenommen wurden.

\section{Literatur}

Balch, Alex, Ivana Fellini, Anna Ferro, Giovanna Fullin, und Uwe Hunger. 2004. The political economy of labour migration in the European construction sector. In Organisational recruitment and patterns 
of migration IMIS-Beiträge 25, Hrsg. Michael Bommes, Kirsten Hoesch, Uwe Hunger, und Holger Kolb, 179-199. Osnabrück: Institut für Migrationsforschung und Interkulturelle Studien.

Barslund, Mikkel, Matthias Busse, und Frederic De Wispelaere. 2017. Posted workers-for some it matters. Centre for European Policy Studies. https://www.ceps.eu/publications/posted-workers-forsome-it-matters. Zugegriffen: 15. Dez. 2017.

Bernaciak, Magdalena. 2012. Social dumping: political catchphrase or threat to labour standards? Working Paper 2012.06. Brüssel: Europäisches Gewerkschaftsinstitut.

Bogner, Alexander, Beate Littig, und Wolfgang Menz. 2005. Experteninterviews: Theorien, Methoden, Anwendungsfelder, 2. Aufl. Wiesbaden: VS.

Bommes, Michael, Kirsten Hoesch, Uwe Hunger, und Holger Kolb (Hrsg.). 2004. Organisational recruitment and patterns of migration. IMIS-Beiträge 25. Osnabrück: Institut für Migrationsforschung und Interkulturelle Studien.

Bosch, Gerhard. 2013. Grenzüberschreitende Arbeitsmärkte und nationale Beschäftigungssysteme in der EU. In Transnationale Vergesellschaftungen, Hrsg. Hans Georg Soeffner, 279-292. Wiesbaden: Springer VS.

Bosch, Gerhard, Danijel Nestic, und László Neumann. 2013. Minimum wage and collective bargaining in the construction industry. In Minimum wages, pay equity, and comparative industrial relations, Hrsg. Damian Grimshaw, 168-193. New York, London: Routledge.

BUAK. 2017a. Herkunft der Arbeitnehmer in österreichischen Betrieben. Bauarbeiter-Urlaubs- und Abfertigungskasse. https://www.buak.at/cms/BUAK/BUAK_1.5/die-buak/statistiken. Zugegriffen: 10. Okt. 2017.

BUAK. 2017b. Statistik Entsendung Betriebe und Arbeitnehmer für die Jahre 2011-2016.

Dickens, Linda. 2012. Fairer workplaces: making employment rights effective. In Making employment rights effective: Issues of compliance and enforcement, Hrsg. Linda Dickens, 205-228. Oxford: Hart Publishing.

Die Presse. 2017. 40 Prozent der Auslandsfirmen am Bau unter Lohndumping-Verdacht. https://diepresse. com/home/wirtschaft/unternehmen/5296837/40-Prozent-der-Auslandsfirmen-am-Bau-unterLohndumpingVerdacht. Zugegriffen: 15. Dez. 2017.

Dølvik, Jon Erik, Line Eldring, und Jelle Visser. 2014. Setting wage floors in open labour markets: the role of the social partners in Europe's multilevel governance. In Regulating transnational labour in europe: the quandaries of multilevel governance, Hrsg. Stein Evju, 131-186. Oslo: University of Oslo, Department of Private Law.

Eichhorst, Werner. 1999. Europäische marktgestaltende Politik zwischen Supranationalität und nationaler Autonomie: Das Beispiel der Entsenderichtlinie. Industrielle Beziehungen 6(3):340-359.

Eppel, Rainer, Thomas Leoni, und Helmut Mahringer. 2017. Österreich 2025: Segmentierung des Arbeitsmarktes und schwache Lohnentwicklung in Österreich. Wien: Österreichisches Institut für Wirtschaftsforschung.

Europäische Kommission. 2012. Posting of workers in the European union and EFTA countries: report on A1 portable documents issued in 2010 and 2011. Brüssel: Generaldirektion Beschäftigung, Soziales und Integration.

Eurostat. 2017. Löhne und Arbeitskosten. http://ec.europa.eu/eurostat/statistics-explained/index.php? title=Wages_and_labour_costs/de. Zugegriffen: 26. Juni 2018.

Fellini, Ivana, Anna Ferro, und Giovanna Fullin. 2007. Recruitment processes and labour mobility: the construction industry in Europe. Work, Employment and Society 21(2):277-298. https://doi.org/10. $1177 / 0950017007076635$.

Flecker, Jörg. 2009. Outsourcing, spatial relocation and the fragmentation of employment. Competition \& Change 13(3):252-268. https://doi.org/10.1179/102452909X451369.

Gagawczuk, Walter. 2018. Die Europäische Arbeitsbehörde - Lohndumping entgegenwirken. infobrief eu \& international 1/2018. Kammer für Arbeiter und Angestellte für Wien. https://media. arbeiterkammer.at/wien/EU_Infobrief_1_2018.pdf. Zugegriffen: 22. Febr. 2018.

Gläser, Jochen, und Grit Laudel. 2009. Experteninterviews und Qualitative Inhaltsanalyse als Instrumente rekonstruierender Untersuchungen, 3. Aufl. Wiesbaden: VS.

Grimshaw, Damian, Colette Fagan, Gail Hebson, und Isabel Tavora. 2017. A new labour market segmentation approach for analysing inequalities: introduction and overview. In Making work more equal: A new labour market segmentation approach, Hrsg. Damian Grimshaw, Colette Fagan, Gail Hebson, und Isabel Tavora, 1-32. Manchester: Manchester University Press.

Haidinger, Bettina. 2016. Grenzenlose Mobilität - Grenzenlose Ausbeutung. Arbeitsbedingungen in Europas Transportwirtschaft. Wien: FORBA/AK. 
Harvey, Mark. 2003. Privatization, fragmentation and inflexible flexibilization in the UK construction industry. In Building chaos: an international comparison of deregulation in the construction industry, Hrsg. Gerhard Bosch, Peter Philips, 188-209. London, New York: Routledge.

Hermann, Christoph, und Jörg Flecker. 2006. Betriebliche Interessenvertretung in Österreich. FORBA Schriftenreihe 1/2006. Wien: Forschungs- und Beratungsstelle Arbeitswelt.

Hofstadler, Christian, Markus Loik, Markus Peterseil, Nenad Pantelic, und Nicholas Katz. 2016. Einfluss von Lohn- und Sozialdumping auf den Wettbewerb in der Bauwirtschaft. Graz: Technische Universität Graz, Institut für Baubetrieb und Bauwirtschaft.

Holst, Hajo. 2015. Europäisierung als institutionelle Entbettung - Finanzialisierte multinationale Konzerne und die Arbeitsbeziehungen im europäischen Paketsektor. In Horizontale Europäisierung im Feld der Arbeitsbeziehungen, Hrsg. Susanne Pernicka, 151-182. Wiesbaden: Springer VS.

Huber, Peter, und Georg Böhs. 2012. Monitoring der Arbeitsmarktauswirkungen der Zuwanderung aus den neuen EU-Mitgliedsländern im Regime der Freizügigkeit - Begleitende Beratung und Analyse. In Arbeitsmarktöffnung 2011 Sozialpolitische Studienreihe Band, Bd. 12, 29-234. Wien: Bundesministerium für Arbeit, Soziales und Konsumentenschutz.

Hunger, Uwe. 2000. Temporary transnational labour migration in an integrating Europe and the challenge to the German welfare state. In Immigration and welfare: Challenging the boundaries of the welfare state, Hrsg. Michael Bommes, Andrew Geddes, 186-204. London: Routledge.

Krings, Torben. 2013. Von der „Ausländerbeschäftigung“ zur Rot-Weiß-Rot-Karte: Sozialpartnerschaft und Migrationspolitik in Österreich. Österreichische Zeitschrift für Politikwissenschaft 42(3):263-278. https://doi.org/10.15203/ozp.123.vol42iss3.

Krings, Torben. 2016. East-West mobility and the (re-)regulation of employment in transnational labour markets. In Labour mobility in the enlarged single European market Comparative Social Research, Bd. 32, Hrsg. Jon Erik Dølvik, Line Eldring, 183-213. Bingley: Emerald Group Publishing.

Pacolet, Jozef, und Frederic De Wispelaere. 2018. Posting of workers: report on A1 portable documents issued in 2016. Brüssel: Europäische Kommission, Generaldirektion Beschäftigung, Soziales und Integration.

Piore, Michael J. 1979. Birds of passage: migrant labor and industrial societies. Cambridge: Cambridge University Press.

Riesenfelder, Andreas, Susanne Schelepa, und Petra Wetzel. 2012. Monitoring der Arbeitsmarktöffnung Auswirkungen auf Beschäftigungsformen und auf Lohndumping. In Arbeitsmarktöffnung 2011 Sozialpolitische Studienreihe, Bd. 12, 235-479. Wien: Bundesministerium für Arbeit, Soziales und Konsumentenschutz.

Rubery, Jill. 2007. Developing segmentation theory: a thirty year perspective. Économies et Sociétés 41(6):941-964.

Scharpf, Fritz W. 1998. Balancing positive and negative integration: the regulatory options for Europe. In The challenge of globalization for Germany's SocialDemocracy. A policy agenda for the twenty-first century, Hrsg. Dieter Dettke, 29-57. Oxford: Berghahn Books.

Schmatz, Susanne, und Petra Wetzel. 2014. Entwicklung im Bereich des Lohndumping. Wien: L\&R Sozialforschung.

Schweighofer, Johannes. 2012. Gab es auf regionalsektoraler Ebene Verdrängungseffekte im Gefolge der Arbeitsmarktöffnung vom Mai 2011? Wirtschaft und Gesellschaft 38(3):601-614.

Sozialministerium. 2018. Datenbank BALI. http://www.dnet.at/bali. Zugegriffen: 14. Juli 2018.

Staples, Ronald, Rainer Trinczek, und Michael Whittall. 2013. Posted Workers: Zwischen Regulierung und Invisibilisierung. Arbeit 22(4):271-286.

Szigetvari, András. 2018a. Starker Anstieg temporärer Arbeitnehmer aus Osteuropa. Der Standard, 9. Febr. 2018.

Szigetvari, András. 2018b. Was gegen Sozialbetrug am Bau helfen könnte. Der Standard, 20. Apr. 2018.

Wagner, Bettina, und Anke Hassel. 2015. Labor migration and the German meat processing industry: fundamental freedoms and the influx of cheap labor. South Atlantic Quarterly 114(1):204-214. https:// doi.org/10.1215/00382876-2831643.

Wagner, Ines, und Nathan Lillie. 2014. European integration and the disembedding of labour market regulation: transnational labour relations at the European Central Bank construction site. Journal of Common Market Studies 52(2):403-419. https://doi.org/10.1111/jcms.12096.

Weil, David. 2010. Improving workplace conditions through strategic enforcement: A report to the wage and hour division. Boston University. https://www.dol.gov/whd/resources/strategicEnforcement.pdf. Zugegriffen: 15. Juli 2018. 
Wiesböck, Laura. 2016. A preferred workforce? Employment practices of East-West cross border labour commuters in the Central European Region. Österreichische Zeitschrift für Soziologie 41(4):391-407. https://doi.org/10.1007/s11614-016-0245-3.

Wiesinger, Christoph. 2015. Elemente der Sozialbetrugsbekämpfung. In Sozialbetrugsbekämpfung in der Bauwirtschaft: Auftraggeberhaftung - Lohn- und Sozialdumping - Ausländerbeschäftigung, Hrsg. Christoph Wiesinger, 1-27. Wien: Linde.

Torben Krings arbeitet als Assoziierter Professor an der Abteilung für Wirtschafts- und Organisationssoziologie der Johannes Kepler Universität Linz. Seine Forschungsinteressen sind Migration, Arbeit und Beschäftigung sowie Europäische Gesellschaften. Jüngere Publikation: East-West mobility and the (re-) regulation of employment in transnational labour markets. In Labour mobility in the enlarged single European market, Hrsg. Jon Erik Dølvik und Line Eldring, 2016. 